\title{
The measurement of left-right asymmetries in the Simon effect: A fine-grained analysis
}

\author{
Mariaelena Tagliabue, Giulio Vidotto, Carlo Umiltà, \\ Gianmarco Altò̀, Barbara Treccani, and Paola Spera \\ University of Padua, Padua, Italy
}

\begin{abstract}
The spatial Simon effect is often asymmetric, being greater on one side than on the other. To date, not much attention has been paid to these asymmetries, and explanations of the Simon effect do not take them into account. In the present article, we attempt to clarify the statistical implications of the asymmetries so as to provide a useful tool for future empirical investigation. Starting with examples from our laboratory and previous well-known studies, we point out the consequences of ignoring the asymmetries in the Simon effect. We suggest an alternative data analysis that might render the results clearer. Finally, through a comparison of left- and right-handed subjects, we demonstrate that asymmetries in the Simon effect are linked to the lateralization of processes involved in the Simon task - that is, attention and response selection. This approach provides a strong argument against collapsing data from the two sides to measure the Simon effect.
\end{abstract}

When a subject must select the left or right response on the basis of a nonspatial feature of the stimulus (e.g., color), reaction time (RT) is shorter when the positions of the stimulus and of the response correspond than when they do not. This is the spatial Simon effect, which is also present when the two responses are executed with two fingers of the same hand (Umiltà \& Nicoletti, 1985) and when the two stimuli are presented in the same visual hemifield (relative left and right positions; Umiltà \& Liotti, 1987). A widely shared assumption (see, e.g., Lu \& Proctor, 1995, for a review) is that this phenomenon is due to a competition, at the response selection stage, between the response spatially corresponding to the stimulus, which is automatically activated by stimulus presentation, and the response selected, in a controlled way, on the basis of the relevant stimulus attribute.

Until some years ago, researchers reported the Simon effect as an interaction between stimulus position and response position. In contrast, now the Simon effect is almost always reported as an overall effect, with corresponding and noncorresponding trials that take place on the two sides collapsed (see, e.g., Masaki, Takasawa, \&Yamazaki, 2000; Proctor \& Lu, 1999; Tagliabue, Zorzi, \& Umiltà, 2002). In other words, the overall Simon effect was indexed in the ANOVA by the two-way response position $\times$ stimulus position interaction, and researchers reported the four mean RTs of the interaction: RT for the right response to the stimulus on the right, RT for the left response to the stimulus on the right, RT for the right response to the stimulus on the left, and RT for the left response to the stimulus on the left. (Henceforth, we will refer to this type of analysis as the Design 1 ANOVA).
Thus, the overall Simon effect was in fact the mean of two lateralized Simon effects - that is, RT for the left response to the stimulus on the right minus RT for the right response to the stimulus on the right, and RT for the right response to the stimulus on the left minus RT for the left response to the stimulus on the left (but see below for an alternative way to describe the two lateralized Simon effects). Now the Simon effect is instead indexed in the ANOVA by the main effect of correspondence (corresponding vs. noncorresponding trials), and researchers report only the overall Simon effect - that is, the mean of the two types of noncorresponding trials (RT for the left response to the stimulus on the right and RT for the right response to the stimulus on the left) minus the mean for the two types of corresponding trials (RT for the right response to the stimulus on the right and RT for the left response to the stimulus on the left).

Thus, the overall Simon effect, on which most of the more recent studies are based, is in fact the algebraic mean of two distinct Simon effects that often differ in size and may or may not differ in significance (i.e., both significant, both nonsignificant, or one significant and one nonsignificant). Actually, distinguishing between the two lateralized Simon effects is considered to be of negligible importance. In this section, we will explain why, in our opinion, it is important to have an index of the asymmetries and will discuss different ways to index them. The main point is to stress the fact that some statistical designs provide information about asymmetries in the Simon effect, whereas others conceal their presence.

Moreover, the asymmetry between the two lateralized Simon effects might provide evidence in favor of one of

M. Tagliabue, mariaelena.tagliabue@unipd.it 
the two main hypotheses that have been put forward to explain why, in the Simon task, the task-irrelevant code of stimulus position is formed. These are the attentional shift hypothesis (Nicoletti \& Umiltà, 1994; Stoffer, 1991; Umiltà \& Nicoletti, 1992) and the referential coding hypothesis (Hommel, 1993; Hommel \& Lippa, 1995). The attentional shift hypothesis claims that stimulus position is automatically coded because of the need to shift attention toward the stimulus. Hence, the (potentially interfering) spatial stimulus code is formed in relation to the position in which attention is focused when the stimulus appears. According to the referential coding hypothesis, the location of the stimulus is coded in relation to its relative position with respect to an object that acts as a reference frame.

Studies (de Gonzaga Gawryszewski, Riggio, Rizzolatti, \& Umiltà, 1987; Downing \& Pinker, 1985; see also Umiltà, 2000, for a review) have demonstrated left-right asymmetries in the mechanisms of spatial attention (orienting). If one assumes that the stimulus spatial code in the Simon task is the result of an attention shift, it is reasonable also to assume that left-right asymmetries in shifting attention might be at the origin of the left-right asymmetries that are found in the Simon effect. In contrast, the referential coding hypothesis would need a posteriori adjustments to account for the left-right asymmetries in the Simon effect. In fact, it is not immediately clear why, if left and right codes are formed in relation to a frame of reference, the left code would take more or less time to be formed than the right code. If left-right asymmetries in the Simon effect might help one discriminate between the attention shift hypothesis and the referential coding hypothesis, it becomes very interesting to find ways to determine when they are present, what their magnitudes are on the two sides, and, of course, whether or not they are statistically reliable.

In brief, we are convinced that left-right asymmetries are not a trivial aspect of the Simon effect and should be taken into consideration by any hypothesis intended to explain the Simon effect. Therefore, it becomes important to establish, in the first place, whether asymmetry is present, not only in new studies but also in previous studies. Unfortunately, in previous studies researchers never (or very seldom) reported asymmetries. One of the goals of the present article is, thus, to propose a method for establishing the presence of asymmetries in previous studies in which asymmetries were not explicitly reported. Another goal is to develop a more informative method for analyzing asymmetries in new studies in which asymmetries are explicitly taken into consideration.

\section{Different Ways to Analyze Data From Simon Tasks}

Before we proceed, however, a further clarification is necessary. There are two ways of describing a Simon effect: one with reference to the stimulus and one with reference to the response. Note that in the previous studies these two ways of describing the Simon effect were never explicitly distinguished but rather used interchangeably. One can keep the stimulus constant and compare the two

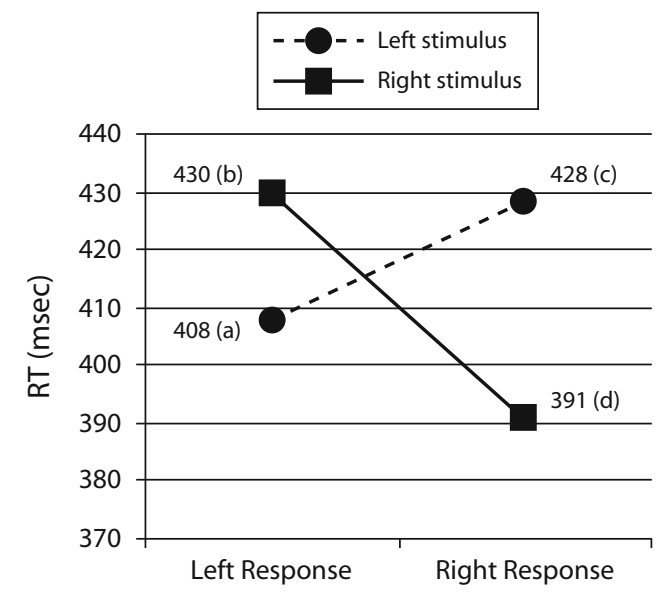

Figure 1. Mean reaction times for the two-way interaction showing the asymmetrical Simon effect of the subjects in Experiments 3, 4, and 5 of Tagliabue, Zorzi, Umiltà, and Bassignani (2000). Letters index the corresponding left response (a), the noncorresponding left response (b), the noncorresponding right response (c), and the corresponding right response (d).

responses (e.g., left response to the right stimulus vs. right response to the right stimulus). Alternatively, one can keep the response constant and compare the two stimuli (e.g., right response to the left stimulus vs. right response to the right stimulus). In what follows, the terms left stimulus/left response, right stimulus/left response, left stimulus/right response, and right stimulus/right response (a, b, c, and $\mathrm{d}$, respectively, in Figure 1) will be used for individual trials, and the terms stSE and reSE will be used for the stimulus and response Simon effects, respectively - that is, the difference between noncorresponding and corresponding trials with reference to one stimulus (e.g., left stSE $=$ right response RT to the left stimulus minus left response RT to the left stimulus, or c - a) or one response (e.g., right reSE = right response RT to the left stimulus minus right response RT to the right stimulus, or $\mathrm{c}-\mathrm{d}$ ). As is shown in Figure 1, asymmetries are present in the empirical data from Tagliabue, Zorzi, Umiltà, and Bassignani (2000), and the size of the Simon effect is different on the two sides. The asymmetry may consist of a smaller Simon effect for the stimulus on one side (stSE, indicated by the slopes of the two lines) or of a smaller Simon effect for the response on one side (reSE, indicated by the differences between the two data points for the left and right responses).

The idea of investigating the asymmetries in the Simon effect originated from inspection of our previously collected data (those shown in Figure 1 serve as an example). ${ }^{1}$ The goal of the original study was to investigate the influence of spatial compatibility tasks on the Simon effect. The data were those reported by Tagliabue et al. (2000), in which asymmetries were clear if we consider only those conditions in which practice did not influence the Simon effect - that is, when the Simon task was performed after the compatible version of the spatial compatibility tasks. In Experiment 1 (in which children served as subjects and stimulus exposure time was long), there was a negative left stSE. In Experiments 2, 3, and 4, the left reSE and the 
Table 1

Equivalence of the Three Designs of ANOVAs Carried Out on

Tagliabue et al.'s (2000) Data, and Orthogonal Planned Comparisons

Based on the Hypothesis That Disregards Asymmetries (Plan 1)

\begin{tabular}{|c|c|c|c|c|c|c|c|c|c|c|}
\hline \multicolumn{3}{|c|}{ ANOVA Design } & \multicolumn{4}{|c|}{$\begin{array}{l}\text { CWs for Equivalent } \\
\text { Planned Comparisons }\end{array}$} & \multicolumn{4}{|c|}{ Results } \\
\hline 1 & $2 \mathrm{~A}$ & $2 \mathrm{~B}$ & $\overline{\mathrm{a}}$ & $\mathrm{b}$ & $\mathrm{c}$ & $\bar{d}$ & $F(1,28)$ & $M S_{\mathrm{e}}$ & $M S$ Effect & $p$ \\
\hline $\bar{A}$ & $\mathrm{~B} \times \mathrm{C}$ & A & 1 & -1 & 1 & -1 & 4.27 & 374.75 & $1,601.61$ & .048058 \\
\hline B & $\mathrm{B}$ & $\mathrm{A} \times \mathrm{C}$ & 1 & 1 & -1 & -1 & 4.34 & 618.24 & $2,682.54$ & .046500 \\
\hline $\mathrm{A} \times \mathrm{B}$ & $\mathrm{C}$ & $\mathrm{C}$ & 1 & -1 & -1 & 1 & 57.58 & 446.29 & $25,698.65$ & .000000 \\
\hline
\end{tabular}

Note-A, stimulus position; B, response position; $\mathrm{C}$, correspondence; $\mathrm{CW}$, contrast weight; a, corresponding left response; $b$, noncorresponding left response; $c$, noncorresponding right response; $d$, corresponding right response.

left stSE were much smaller than the right reSE and the right stSE, respectively. In Experiments 5 and 6, the left stSE was smaller than the right stSE.

To ascertain that the asymmetries were reliable, we reanalyzed the data from 29 subjects of Tagliabue et al.'s (2000) Experiments 3, 4, and 5 who had performed the Simon task after the spatially compatible task. ${ }^{2}$ The three experiments had comparable experimental conditions. The ANOVA on RTs, with stimulus position and response position as the two within-subjects factors (Design 1 ANOVA), showed significant main effects of stimulus position, with mean RTs of $418 \mathrm{msec}$ for the left stimulus and $410 \mathrm{msec}$ for the right stimulus $[F(1,28)=4.27$, $M S_{\mathrm{e}}=374.75, M S$ effect $\left.=1,601.61, p<.05\right]$, and of response position, with RTs of $419 \mathrm{msec}$ for the left response and $409 \mathrm{msec}$ for the right response $[F(1,28)=$ $4.34, M S_{\mathrm{e}}=618.24, M S$ effect $\left.=2,682.54, p<.05\right]$. The interaction also reached significance $[F(1,28)=$ $57.58, M S_{\mathrm{e}}=446.29, M S$ effect $\left.=25,698.65, p<.001\right]$. (Pooled mean RTs for the three experiments are shown in Figure 1.) The Simon effect was always present bilaterally, but the left stSE was smaller than the right stSE (20 vs. $39 \mathrm{msec}$ ) and the left reSE was smaller than the right reSE (22 vs. $37 \mathrm{msec}$ ). Unfortunately, we were not able to determine whether or not these asymmetries were statistically reliable.

In fact, in the Design 1 ANOVA the significance of left and right reSEs or of left and right stSEs is usually tested with the Newman-Keuls post hoc test. However, the use of post hoc tests is questionable because they lack statistical power. The reason is that, because several post hoc tests assess the same null hypothesis, the likelihood that one of them will yield a Type I error is higher than the critical alpha value chosen. To overcome this problem, the alpha level associated with each comparison must be lowered. That, of course, decreases the likelihood that each comparison will achieve significance and thus reduces statistical power (Keppel, 1991). Another undesirable consequence is that different post hoc tests might produce different results. In addition, post hoc tests are informative about asymmetries when the Simon effect is nonsignificant on one side, but they tell us nothing when the left and right Simon effects are different in size but both significant.

One might think that a different ANOVA with two within-subjects factors - that is, correspondence and response position (Design 2A ANOVA) or, alternatively, correspondence and stimulus position (Design 2B ANOVA) - would provide more information about asymmetries. However, this reasoning is unsound because the only difference between these analyses and the previous ones (Design 1) is in the order in which the variables are arranged. As is shown in Table 1, which reports the results of different ANOVA designs on Tagliabue et al.'s (2000) data, the values of the Design 1 ANOVA (with stimulus position and response position $[\mathrm{A} \times \mathrm{B}]$ as the withinsubjects factors) are the same as those of the Design 2A (with response position and correspondence $[\mathrm{B} \times \mathrm{C}]$ as the within-subjects factors) and Design $2 \mathrm{~B}$ (with stimulus position and correspondence $[\mathrm{A} \times \mathrm{C}]$ as the within-subjects factors) ANOVAs. The statistical values of Factor A in the Design 1 and Design 2B ANOVAs are the same as those of the $\mathrm{B} \times \mathrm{C}$ interaction in the Design 2A ANOVA. The statistical values of Factor B in the Design 1 and Design 2A ANOVAs are the same as those of the $\mathrm{A} \times \mathrm{C}$ interaction in the Design 2B ANOVA. The statistical values of the $\mathrm{A} \times \mathrm{B}$ interaction of the Design 1 ANOVA - which indicate that the Simon effect is present - are the same as those of the $\mathrm{C}$ factor in the other two ANOVA designs. In other words, all these ANOVA designs are equivalent. Note that they are all based on interactions; thus, we will group these analyses under the name of interaction-based ANOVAs.

A third method for assessing asymmetries in the Simon effect is to compare, in separate ANOVAs, the left versus the right reSE and the left versus the right stSE. Thus, we carried out two ANOVAs on the data of Tagliabue et al. (2000), one with reSE as the dependent variable (Design $3 \mathrm{~A}$ ) and the other with stSE as the dependent variable (Design 3B). For Design 3A, the one within-subjects factor was side of reSE (left vs. right), and for Design 3B it was side of stSE (left vs. right). The ANOVA on reSE showed a significant main effect of side, with $22 \mathrm{msec}$ for the left reSE versus $37 \mathrm{msec}$ for the right $\operatorname{reSE}[F(1,28)=$ $4.27, M S_{\mathrm{e}}=749.49, M S$ effect $\left.=3,203.22, p<.05\right]$, thus confirming that the left reSE was significantly smaller than the right. Similarly, the ANOVA on stSE showed a significant main effect of side, with $20 \mathrm{msec}$ for the left stSE versus $39 \mathrm{msec}$ for the right stSE $[F(1,28)=4.34$, $M S_{\mathrm{e}}=1,236.49, M S$ effect $\left.=5,365.07, p<.05\right]$, thus also confirming that the left stSE was significantly smaller than the right stSE. 


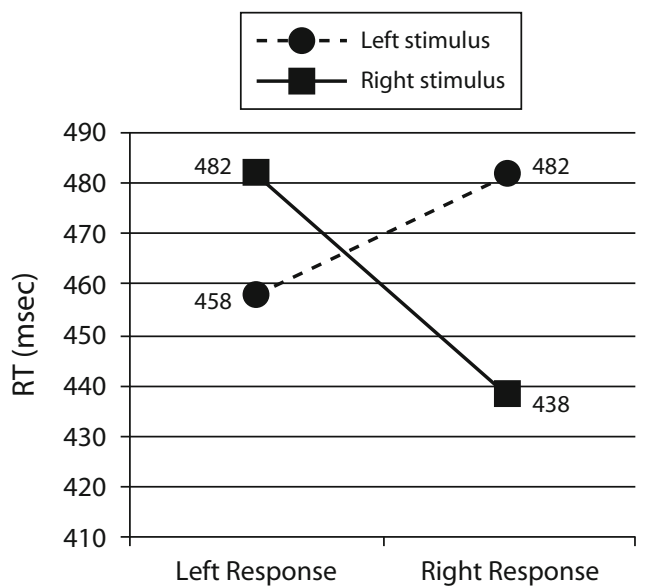

Figure 2. Mean reaction times for the two-way interaction showing the asymmetrical Simon effect of the subjects in Experiments 1, 2, and 3 of Tagliabue et al. (2002).

It is worth noting that the treatment mean square $(M S$ effect) and the within-groups mean square $\left(M S_{\mathrm{e}}\right)$ of the reSE factor in the Design 3A ANOVA are twice as large as the $M S$ effect and $M S_{\mathrm{e}}$ of Factor A (stimulus position) in the Design 1 ANOVA. Similarly, the $M S$ effect and $M S_{\text {e }}$ of the stSE factor in the Design 3B ANOVA are twice as large as the $M S$ effect and $M S_{\mathrm{e}}$ of Factor B (response position) in the Design 1 ANOVA; of course, $F$ ratios and $p$ values are identical. Note that the reSE asymmetry is reflected by the stimulus position factor and the stSE asymmetry is reflected by the response position factor. In fact, there is a precise relation between the marginal means of stimulus position and those of the reSE and between the marginal means of response position and those of the stSE, which accounts for the identical $F$ ratios and $p$ values in the ANOVAs. The explanation is as follows. The difference between the right and left stSE is twice as large as the difference between the RTs for the left and right response, and the difference between the right and left reSE is twice as large as the difference between the RTs to the left and right stimulus. In other words, a shorter mean RT for a response on one side is necessarily accompanied by a greater stSE on the same side. Similarly, a shorter mean RT to a stimulus on one side is necessarily accompanied by a greater reSE on the same side. Thus, Design 1, Design 2, and Design 3 ANOVAs are all equivalent. Therefore, on the basis of the significance of the difference between RTs for the stimulus position or response position factors in interaction-based ANOVAs, it is possible to establish whether or not the asymmetries in the Simon effect are significant.

The statistical equivalences discussed thus far are important for two reasons: first, because they provide information about asymmetries from analyses that were not planned for this purpose and, second, because they should alert one to the risks inherent in collapsing data from the left and right sides. In fact, a statistical index of asymmetries in Design 1, Design 2, and Design 3 ANOVAs would be beneficial. In contrast, no information on possible asymmetries is available in the case of ANOVAs in which response position and stimulus position are collapsed into a single factor.

\section{An Overview of the Literature}

At this point, it appeared crucial to ascertain that the data set upon which we based the reasoning outlined above, and which showed clear evidence of asymmetries, was not atypical. Thus, we had to find evidence of asymmetries in additional available data.

An example of a study in which response and stimulus position were collapsed into a single factor is Tagliabue et al. (2002). Because this way of analyzing data does not provide information about asymmetries, we submitted the data from the visual Simon task performed after a spatially compatible acoustic task to a Design 1 ANOVA and, indeed, again found reliable asymmetries. In the ANOVAs on the data of the three experiments taken together, with stimulus position and response position as within-subjects factors, both main effects reached significance. Mean RTs and results of the analyses are shown in Figure 2 and Table 2 , respectively.

Overall, the Simon effect is present as attested by the significance of the interaction in the Design 1 ANOVA or by that of the factor correspondence in the Design 2 ANOVAs. In addition, on the basis of the statistical equivalence previously shown, the significance of stimulus position $\left[F(1,47)=9.54, M S_{\mathrm{e}}=484.59, M S\right.$ effect $=$ $4,620.90, p<.01]$ indicates that the left reSE is significantly different from the right reSE ( $24 \mathrm{vs} .44 \mathrm{msec}$ ), and the significance of response position $[F(1,47)=8.81$, $M S_{\mathrm{e}}=509.02, M S$ effect $\left.=4,483.21, p<.01\right]$ indicates that the left stSE is significantly different from the right stSE ( 24 vs. $44 \mathrm{msec}$ ). Once again, the left Simon effect was smaller for both the stimulus and the response.

Table 2

Equivalence of the Three Designs of ANOVAs Carried Out on

Tagliabue et al.'s (2002) Data, and Orthogonal Planned Comparisons Based on the Hypothesis That Disregards Asymmetries (Plan 1)

\begin{tabular}{|c|c|c|c|c|c|c|c|c|c|c|}
\hline \multicolumn{3}{|c|}{ ANOVA Design } & \multicolumn{4}{|c|}{$\begin{array}{l}\text { CWs for Equivalent } \\
\text { Planned Comparisons }\end{array}$} & \multicolumn{4}{|c|}{ Results } \\
\hline 1 & $2 \mathrm{~A}$ & $2 \mathrm{~B}$ & $\mathrm{a}$ & $\mathrm{b}$ & $\mathrm{c}$ & $\mathrm{d}$ & $F(1,47)$ & $M S_{\mathrm{e}}$ & $M S$ Effect & $p$ \\
\hline $\mathrm{A}$ & $\mathrm{B} \times \mathrm{C}$ & A & 1 & -1 & 1 & -1 & 9.54 & 484.59 & $4,620.90$ & .003377 \\
\hline B & $\mathrm{B}$ & $\mathrm{A} \times \mathrm{C}$ & 1 & 1 & -1 & -1 & 8.81 & 509.02 & $4,483.21$ & .004707 \\
\hline $\mathrm{A} \times \mathrm{B}$ & $\mathrm{C}$ & $\mathrm{C}$ & 1 & -1 & -1 & 1 & 101.60 & 550.69 & $55,949.32$ & .000000 \\
\hline
\end{tabular}

Note-For an explanation of the abbreviations, see Table 1 . 


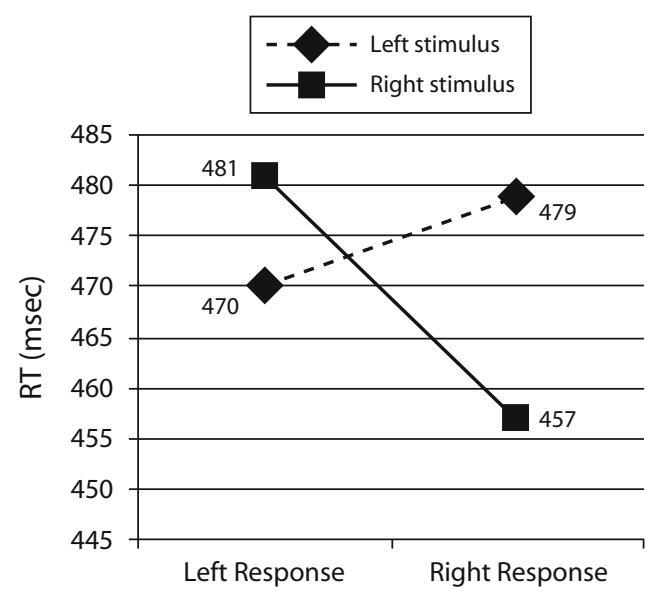

Figure 3. Mean reaction times for the two-way interaction showing the asymmetrical Simon effect of the subjects in the unpublished experiment that replicates Tagliabue et al.'s (2002; Tagliabue et al., 2000) conditions, who performed the spatially compatible task in the tactual modality.

Fortunately, the left-right asymmetries did not affect the main findings of either Tagliabue et al. (2000) or Tagliabue et al. (2002). This is because the Simon effect was normal in size also on the side where it was smaller, and it was significant regardless of the post hoc test employed (LSD, Scheffé, Newman-Keuls, Duncan, or Tukey). However, often the results are more ambiguous. In an unpublished experiment from our laboratory, three groups of 16 subjects performed the visual Simon task 5 min, 1 day, or 1 week after a spatially compatible tactual task, other experimental conditions being equal to those of Tagliabue et al. (2002; Tagliabue et al., 2000). When we submitted the data of the three groups pooled together to the Design 1 ANOVA, both stimulus position $[F(1,47)=$ $3.40, M S_{\mathrm{e}}=393.42, M S$ effect $\left.=1,339.01, p=.07\right]$ and response position $\left[F(1,47)=2.92, M S_{\mathrm{e}}=859.08, M S\right.$ effect $=2,506.35, p=.09]$ approached significance, indicating a smaller left than right reSE (11 vs. $21 \mathrm{msec})$ and a smaller left than right stSE (9 vs. $23 \mathrm{msec}$ ). Mean RTs and results of the analyses are shown in Figure 3 and Table 3A. (The same data are later reanalyzed using orthogonal planned comparisons, and the results are presented in Table 3B; see the section on orthogonal planned comparisons, below.)
Thus, although the interaction between stimulus position and response position was also significant $[F(1,47)=$ $18.03, M S_{\mathrm{e}}=687.25, M S$ effect $\left.=12,393.18, p<.001\right]$, we wondered whether or not the 9- and 11-msec left reSEs were in fact regular and significant Simon effects. Not surprisingly, the Newman-Keuls post hoc test showed them not to be significant ( $p \mathrm{~s}=.11$ and .12 , respectively). Moreover, the left reSE was significant with the LSD post hoc test ( $p=.049)$ and marginally significant with the Duncan post hoc test $(p=.06)$.

Under these circumstances, it is not clear whether the Simon effect is really present or perhaps limited to only one side. What should one conclude about the overall Simon effect? This is a crucial issue, especially if we consider that it would have gone unnoticed had we calculated the overall Simon effect without distinguishing between left and right sides. In our opinion, in interpreting the effect of experimental manipulations, the absence of the Simon effect on one side should not be overlooked.

Other examples of this ambiguity are present in the literature. For instance, in the often cited article by Hedge and Marsh (1975), in which both the stimulus and the response had two dimensions, one spatial and the other nonspatial, ${ }^{3}$ data showed a negative but small left stSE (about $-8 \mathrm{msec}$ ) and a positive right stSE (about $55 \mathrm{msec}$ ). ${ }^{4} \mathrm{In}$ fact, the crucial interaction between stimulus position and response position only approached significance. The authors explained this result by claiming that it was due to the influence of two other factors: practice and order. After these two factors were included in the analysis, a highly significant response position $\times$ stimulus position interaction was obtained. It is clear, however, that the absence of the left stSE weakens the conclusions based on the overall Simon effect.

Another example of this potential problem is provided by the same-color condition of Simon, Sly, and Vilapakkam's (1981) Experiment 2, in which Hedge and Marsh's (1975) condition was replicated with corresponding and noncorresponding trials intermixed. From a look at the figures, it appears that the Simon effect differed in size in the two visual fields (approximately $-12 \mathrm{msec}$ for the left stSE vs. approximately $146 \mathrm{msec}$ for the right stSE). The reSE asymmetry was also present ( $9 \mathrm{msec}$ for the left reSE vs. $125 \mathrm{msec}$ for the right reSE). In brief, in Simon et al. (1981), both asymmetries were present, yet they were not even reported. In discussing their results, the authors ig-

Table 3A

Equivalence of the Three Designs of ANOVAs Carried Out on Unpublished Data That Replicate Tagliabue et al.'s (2002; Tagliabue et al., 2000) Conditions With the Spatially Compatible Task in the Tactual Modality, and Orthogonal Planned Comparisons Based on the Hypothesis That Disregards Asymmetries (Plan 1)

\begin{tabular}{|c|c|c|c|c|c|c|c|c|c|c|}
\hline \multicolumn{3}{|c|}{ ANOVA Design } & \multicolumn{4}{|c|}{$\begin{array}{l}\text { CWs for Equivalent } \\
\text { Planned Comparisons }\end{array}$} & \multicolumn{4}{|c|}{ Results } \\
\hline 1 & $2 \mathrm{~A}$ & $2 \mathrm{~B}$ & $\mathrm{a}$ & $\mathrm{b}$ & $\mathrm{c}$ & d & $F(1,47)$ & $M S_{\mathrm{e}}$ & $M S$ Effect & $p$ \\
\hline $\mathrm{A}$ & $\mathrm{B} \times \mathrm{C}$ & $\mathrm{A}$ & 1 & -1 & 1 & -1 & 3.40 & 393.42 & $1,339.01$ & .071366 \\
\hline B & B & $\mathrm{A} \times \mathrm{C}$ & 1 & 1 & -1 & -1 & 2.92 & 859.08 & $2,506.35$ & .094223 \\
\hline $\mathrm{A} \times \mathrm{B}$ & $\mathrm{C}$ & $\mathrm{C}$ & 1 & -1 & -1 & 1 & 18.03 & 687.25 & $12,393.18$ & .000102 \\
\hline
\end{tabular}

Note-A, B, and C indicate independent variables, whereas the letters a, b, c, and d indicate the four conditions of the Simon task (see Table 1). CW, contrast weight. 
Table 3B

A Method of Variance Partitioning for Orthogonal Planned Comparisons

Based on the Hypothesis That Considers Asymmetries in the Simon Effects, Performed on Unpublished Data That Replicate Tagliabue et al.'s (2002; Tagliabue et al., 2000) Conditions

\begin{tabular}{|c|c|c|c|c|c|c|c|c|}
\hline \multirow[b]{2}{*}{ ANOVA Design } & \multicolumn{4}{|c|}{$\begin{array}{l}\text { CWs for Planned } \\
\text { Comparisons }\end{array}$} & \multicolumn{4}{|c|}{ Results } \\
\hline & $\mathrm{a}$ & $\mathrm{b}$ & $\mathrm{c}$ & d & $F(1,47)$ & $M S_{\mathrm{e}}$ & $M S$ Effect & $p$ \\
\hline \multicolumn{9}{|l|}{ Plan 2} \\
\hline Left reSE & -1 & 1 & 0 & 0 & 5.25 & 531.92 & $2,792.45$ & .026478 \\
\hline Right reSE & 0 & 0 & 1 & -1 & 19.94 & 548.76 & $10,939.74$ & .000050 \\
\hline $\mathrm{B}$ or stSE* & 1 & 1 & -1 & -1 & 2.92 & 859.08 & $2,506.35$ & .094223 \\
\hline \multicolumn{9}{|l|}{ Plan 3} \\
\hline Left stSE & -1 & 0 & 1 & 0 & 2.58 & 727.22 & $1,876.47$ & .114899 \\
\hline Right stSE & 0 & 1 & 0 & -1 & 15.90 & 819.11 & $13,023.07$ & .000232 \\
\hline A or reSE* & 1 & -1 & 1 & -1 & 3.40 & 393.42 & $1,339.01$ & .071366 \\
\hline
\end{tabular}

Note- A and B indicate independent variables, whereas the letters a, b, c, and d indicate the four conditions of the Simon task (see Table 1). CW, contrast weight; left reSE, Simon effect for left responses; right reSE, Simon effect for right responses; left stSE, Simon effect for left stimuli; right stSE, Simon effect for right stimuli. ${ }^{*} M S_{\mathrm{e}}$ and $M S$ effect halved.

nored the negative left stSE and the small, perhaps null, left reSE. Thus, their conclusions about the overall Simon effect are uncertain.

Although a review of the literature is beyond the scope of the present work, it is worth noting that other well-known articles show evidence of asymmetries. For instance, asymmetries were present in the studies by Simon (1968) and Simon and Rudell (1967), in which acoustic stimuli consisted of the words left and right presented to the left or to the right ear and participants had to respond on the basis of the meaning of the word stimulus. A greater Simon effect on the right than on the left was also shown in the studies by Simon, Craft, and Webster (1973) and Simon, Small, Ziglar, and Craft (1970), in which tones of different frequencies were presented to the left or the right ear and responses had to be selected on the basis of the pitch of the tone.

Asymmetries were also present when visual stimuli were used. This was the case, for instance, in Craft and Simon (1970), in which subjects were asked to respond with one of the two hands depending on the color of the stimulus light, and in Wallace (1971), in which the stimuli were either squares or circles and the response depended on the shape of the stimulus.

In those studies, asymmetries always tended toward a smaller left Simon effect. In contrast, examples of studies that showed an apparent reverse pattern of asymmetries include those of Callan, Klisz, and Parsons (1974) and Hommel $(1993,1996)$. This renders it even more crucial to be able to ascertain the presence and direction of asymmetries.

\section{When Asymmetries Weaken Data Interpretation}

It should be evident at this point that, at least in some well-known and influential articles, asymmetries are present in the Simon effect and are often in the direction of a smaller left than right Simon effect. The left Simon effect can be large enough to be considered present or small enough (negative or close to 0) to be considered null. Undoubtedly, in the latter case much caution is needed in interpreting the results. In addition, there are cases in which it is difficult to decide whether the left Simon effect manifested itself or not. This can lead to an inability to interpret the results even when sample size is considered "adequate" in the literature on the Simon effect.

\section{EXPERIMENT 1}

The experiment described here belongs to a different project. The goal of the project was to investigate whether or not performing a Simon task in which the response was to be selected on the basis of a nonspatial feature of the stimulus (e.g., color) influences performance on a subsequent Simon task in which the response depended on a different nonspatial feature (e.g., shape). Thus, a group of subjects performed a Simon task with color as the relevant stimulus feature; then, after a brief rest, the same subjects performed a Simon task in which the relevant stimulus feature was shape. Finally, they again performed the Simon task based on color. For color, we found an overall 17-msec Simon effect, which did not interact with session. In what follows, we reanalyze the data from the first session to show how disregarding asymmetries may conceal interesting aspects of the results.

\section{Method}

Subjects. Twenty students of the University of Padua (2 male and 18 female, 19-24 years of age) participated in the experiment. All were right-handed, had normal or corrected-to-normal vision, and were unaware of the purpose of the experiment.

Apparatus and Stimuli. The subjects were seated in front of an IBM 386 computer connected to an NEC MultiSync 3FG color monitor through a VGA card. The experiment was run using the Micro Experimental Laboratory (MEL, Version 2.0) software system. A central $0.7^{\circ} \times 0.7^{\circ}$ cross was presented at fixation, accompanied by a $440-\mathrm{Hz}$ warning tone. Target stimuli were two colored squares (green and red) approximately $1.5^{\circ} \times 1.5^{\circ}$ in size, shown $10^{\circ}$ to the right and to the left of fixation. Responses were executed by pressing the F key (left of body midline) or the K key (right of body midline) with the left or right index finger, respectively. Both keys were covered by white stickers.

Procedure. All the subjects performed a Simon task. Trials began with the presentation of the fixation cross, which remained visible for $1,800 \mathrm{msec}$. The acoustic warning tone was delivered at the onset 


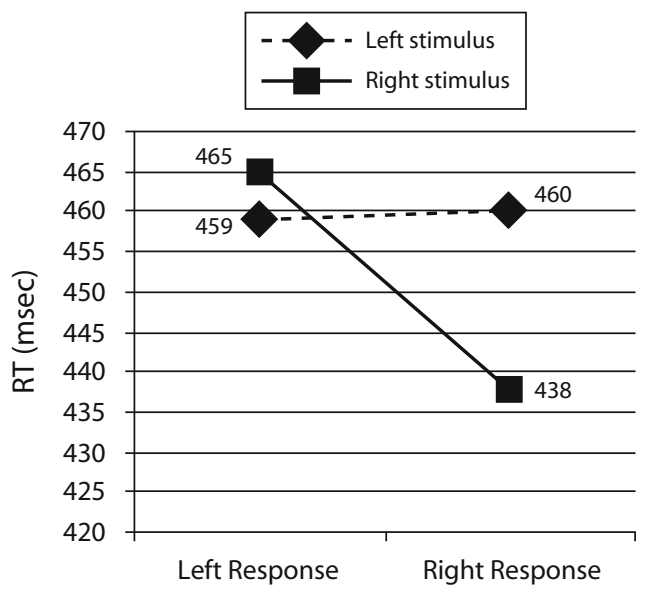

Figure 4. Mean reaction times for the two-way interaction showing the asymmetrical Simon effect of the group of subjects in Experiment 1 of the present study.

of fixation and lasted for $300 \mathrm{msec}$. At the offset of fixation, the imperative stimulus appeared for $100 \mathrm{msec}$. After 1,700 msec, a new trial began. The maximum allowed response time was $1,500 \mathrm{msec}$.

There was a total of 144 experimental trials, equally distributed across the four stimulus position $\times$ response position conditions and presented in random order. The experimental trials were preceded by as many practice trials as were needed to execute 10 correct trials.

Half of the subjects were instructed to press the right key in response to the red square and the left key in response to the green square. The opposite mapping was assigned to the other half of the subjects.

\section{Results and Discussion}

Correct RTs (excluding anticipations-i.e., RTs shorter than $150 \mathrm{msec}$ ) were submitted to an ANOVA with two between-subjects factors (stimulus position and response position). Mean RTs and results of the analyses are shown in Figure 4 and Table 4A.

Both main factors approached significance [stimulus position: $F(1,19)=3.81, M S_{\mathrm{e}}=332.95, M S$ effect $=$ $1,267.71, p=.066$; response position: $F(1,19)=4.17$, $M S_{\mathrm{e}}=822.51, M S$ effect $\left.=3,429.32, p=.055\right]$. Surprisingly, the crucial interaction also only approached significance $\left[F(1,19)=3.98, M S_{\mathrm{e}}=948.93, M S\right.$ effect $=$ $3,773.55, p=.061]$.

In the ANOVA on errors, which was identical to the previous ANOVA, no source reached significance. Overall, the subjects' error rate was $2 \%$.
Overall, there was a small (14-msec) Simon effect. However, the Simon effect was markedly asymmetrical. As is shown in Figure 4, the left stSE effect was null $(1 \mathrm{msec})$ whereas the right stSE effect was of $27 \mathrm{msec}$. Similarly, the left reSE was very small $(6 \mathrm{msec})$ whereas the right reSE was of $22 \mathrm{msec}$.

What conclusions can be drawn about the Simon effect? Following our reasoning, and on the basis of mean RTs, it might be concluded that the Simon effect is present, as is often the case, only on the right side. However, these data are even more problematic because we have no statistical confirmation of the overall Simon effect. As we have already emphasized in Tables $1-4 \mathrm{~A}$, it is useless to perform other types of interaction-based ANOVA designs because they would provide no additional information.

In the face of results that apparently defy a convincing interpretation, such as those just reported, an alternative method of analysis is needed to establish whether asymmetries are in fact present and reliable. Such a method is outlined below. We will consider only RT data because the ANOVA on errors did not show any effect.

\section{Orthogonal Planned Comparisons:}

\section{An Alternative Method of Variance Partitioning}

One way to summarize equivalences between different ANOVA designs is through planned comparisons. If we consider only the overall Simon effect, disregarding asymmetries, we can define a set of contrast weights (CWs) for mutual orthogonal comparisons (see CWs for planned comparisons, Plan 1, in Tables 1, 2, 3A, and 4A), which will produce the same results as the different ANOVA designs discussed above.

It is worth noting that the results of orthogonal planned comparisons based on the hypothesis of an overall symmetric Simon effect are the same as those obtained with the ANOVA and reported above in the present Results and Discussion section.

However, planned comparisons also provide a further method for directly investigating the hypothesis of asymmetries. In effect, we have already stated that the interaction-based ANOVA is not really useful for this purpose. The direct ANOVA on stSE or reSE (Design 3) also does not provide additional information about asymmetries. If we had predicted the possible presence of asymmetries, the best way to analyze the data would have been not to collapse the data from the two sides, but rather to

Table 4A

Equivalence of the Three Designs of ANOVAs Carried Out on Data From the Present Experiment 1, and Orthogonal Planned Comparisons Based on the Hypothesis That Disregards Asymmetries (Plan 1)

\begin{tabular}{|c|c|c|c|c|c|c|c|c|c|c|}
\hline \multicolumn{3}{|c|}{ ANOVA Design } & \multicolumn{4}{|c|}{$\begin{array}{l}\text { CWs for Equivalent } \\
\text { Planned Comparisons }\end{array}$} & \multicolumn{4}{|c|}{ Results } \\
\hline 1 & $2 \mathrm{~A}$ & $2 \mathrm{~B}$ & $\mathrm{a}$ & $\mathrm{b}$ & $\mathrm{c}$ & $\mathrm{d}$ & $F(1,19)$ & $M S_{\mathrm{e}}$ & $M S$ Effect & $p$ \\
\hline A & $\mathrm{B} \times \mathrm{C}$ & A & 1 & -1 & 1 & -1 & 3.81 & 332.95 & $1,267.71$ & .065927 \\
\hline B & B & $\mathrm{A} \times \mathrm{C}$ & 1 & 1 & -1 & -1 & 4.17 & 822.51 & $3,429.32$ & .055294 \\
\hline $\mathrm{A} \times \mathrm{B}$ & $\mathrm{C}$ & $\mathrm{C}$ & 1 & -1 & -1 & 1 & 3.98 & 948.93 & $3,773.55$ & .060688 \\
\hline
\end{tabular}

Note- A, B, and C indicate independent variables, whereas the letters $a, b, c$, and d indicate the four conditions of the Simon task (see Table 1). CW, contrast weight. 
Table 4B

A Method of Variance Partitioning for Orthogonal Planned Comparisons

Based on the Hypothesis That Considers Asymmetries in the Simon Effects, Performed on Data From the Present Experiment 1

\begin{tabular}{|c|c|c|c|c|c|c|c|c|}
\hline \multirow[b]{2}{*}{ ANOVA Design } & \multicolumn{4}{|c|}{$\begin{array}{l}\text { CWs for Planned } \\
\text { Comparisons }\end{array}$} & \multicolumn{4}{|c|}{ Results } \\
\hline & $\mathrm{a}$ & $\mathrm{b}$ & $\mathrm{c}$ & $\mathrm{d}$ & $F(1,19)$ & $M S_{\mathrm{e}}$ & $M S$ Effect & $p$ \\
\hline \multicolumn{9}{|l|}{ Plan 2} \\
\hline Left reSE & -1 & 1 & 0 & 0 & 0.66 & 506.28 & 333.45 & .427097 \\
\hline Right reSE & 0 & 0 & 1 & -1 & 6.07 & 775.61 & $4,707.81$ & .023465 \\
\hline $\mathrm{B}$ or stSE* & 1 & 1 & -1 & -1 & 4.17 & 822.51 & $3,429.32$ & .055294 \\
\hline \multicolumn{9}{|l|}{ Plan 3} \\
\hline Left stSE & -1 & 0 & 1 & 0 & 0.01 & 773.88 & 4.12 & .942630 \\
\hline Right stSE & 0 & 1 & 0 & -1 & 7.22 & 997.56 & $7,198.76$ & .014614 \\
\hline A or reSE* & 1 & -1 & 1 & -1 & 3.81 & 332.95 & $1,267.71$ & .065927 \\
\hline
\end{tabular}

Note-A and B indicate independent variables, whereas the letters a, b, c, and d indicate the four conditions of the Simon task (see Table 1). CW, contrast weight; left reSE, Simon effect for left responses; right reSE, Simon effect for right responses; left stSE, Simon effect for left stimuli; right stSE, Simon effect for right stimuli. ${ }^{*} M S_{\mathrm{e}}$ and $M S$ effect halved.

investigate the consequences of the experimental manipulations separately for the left and the right Simon effects. In other words, we can perform the planned comparisons as an alternative to the interaction-based ANOVA - that is, without reference to the significance of the effects of the ANOVA.

Thus, in our opinion, the best way to proceed is to focus in turn on the stSE and on the reSE through orthogonal planned comparisons in order to assess separately the left and right Simon effects. The next step is to specify the set of CWs for such comparisons. These are reported in Table 4B, with the results of their applications on the data from the present experiment. Note that the difference between the Plan 1, Plan 2, and Plan 3 comparisons (Tables 4A and 4B) lies in the way in which the total sum of squares of the dependent variable is partitioned: Partitioning is based on the hypothesis tested - that is, the hypothesis of an overall symmetric Simon effect (Plan 1), the hypothesis of a Simon effect asymmetric in relation to response side (reSE, Plan 2), or the hypothesis of a Simon effect asymmetric in relation to stimulus side (stSE, Plan 3).

As can be noted, the results are quite different from those of the ANOVAs in that they demonstrate that the Simon effect is present only on the right side, for both the reSE and the stSE. The lack of significance of the left Simon effect is clearly the reason for which the crucial interaction in the interaction-based ANOVA failed to reach significance. Note that the third $\mathrm{CW}$ of Plan 2 provides exactly the same $F$ and $p$ values as those of response position (Factor B) in the Design 1 ANOVA. Similarly, the third CW of Plan 3 provides exactly the same $F$ and $p$ values as those of stimulus position (Factor A) in the Design 1 ANOVA. Thus, taken together, Plans 2 and 3 provide both the information supplied by the interaction-based ANOVA and additional information about asymmetries.

As we have already pointed out, the difference between this method and the widely used interaction-based ANOVA is the way in which variance is partitioned, which in turn determines the independence between information provided by each comparison. The principle of orthogonality - that is, of independence of informationis the reason that the method of planned comparisons provides additional, and more precise and reliable, information (Keppel, 1991).

Those data concerning the unpublished experiment from our laboratory, in which 48 subjects performed the visual Simon task after performing a spatially compatible tactual task (see Tagliabue et al., 2002, for similar experimental conditions) and which was previously mentioned as an example of results difficult to interpret, are reanalyzed with orthogonal planned comparisons. Results are reported in Table 3B.

The Design 1 ANOVA demonstrated that the left stSE and the left reSE were of 9 and $11 \mathrm{msec}$, respectively. Even though the overall Simon effect was significant, as the significance of the stimulus position $\times$ response position interaction attests, post hoc tests yielded discordant results for the significance of the two left Simon effects. In contrast, planned comparisons based on the hypothesis of asymmetries (Plans 2 and 3) unambiguously showed that the reSE was significant on both the left and the right sides, whereas the stSE was significant only on the right side.

\section{Toward an Explanation of Asymmetries in the Simon Effect}

Once a method for measuring asymmetries was established, we were able to investigate the causes of the asymmetries. In our opinion, asymmetries might be due to the lateralization of processes involved in the Simon task - namely, attention and response selection. The accepted notion is that the Simon effect is due to an interference that occurs at the response selection stage (see, e.g., Lu \& Proctor, 1995). In addition, according to the hypothesis of Umiltà and Nicoletti (1992), which states that the task-irrelevant spatial stimulus feature is coded when an attention shift toward the stimulus is performed, attentional mechanisms also play a crucial role (see also Stoffer, 1991). Therefore, one can ask whether asymmetries in the stSE and the reSE are to be ascribed to the stage in which response is selected and/or to a stage related to attentional processes. 
Indeed, there is evidence of cerebral dominance for both selection of action and attentional control. For instance, Schluter, Krams, Rushworth, and Passingham (2001) demonstrated left cerebral dominance for the response selection process, whereas Downing and Pinker (1985) and de Gonzaga Gawryszewski et al. (1987), considering attentional processes, showed an asymmetric pattern in attentional shifting. Moreover, Rushworth, Nixon, Renowden, Wade, and Passingham (1997) demonstrated not only that a mechanism of covert attention associated with the preparation of limb movements (similar to what happens with eye movements) exists, but also that this mechanism is located in the left hemisphere (in righthanded subjects).

On the basis of the findings of Rushworth et al. (1997), which point to the existence of areas in the left parietal cortex specialized for motor attention, and those of Schluter et al. (2001), which demonstrate the dominance of the left hemisphere for the response selection process in general, it might be hypothesized that asymmetries in the Simon effect are due to left cerebral dominance for motor attention and/or response selection processes. If this were so, asymmetries should be different in left-handed and righthanded subjects. Note that the results of both Rushworth et al. and Schluter et al. were obtained with right-handed subjects. If a difference in asymmetries between left- and right-handed subjects emerged, then the likelihood that those asymmetries were due to the laterality of the processes involved would increase. We expected to see in left-handers a reversal of the asymmetries shown by righthanded subjects. However, a more symmetric Simon effect in left-handers might be interesting too, because very often cerebral dominance is not reversed in left-handers (Bradshaw, 1989).

Note that here the involvement of attention is not understood in terms of a general phenomenon related to an orienting bias due to the habit of reading from left to right. Rather, we refer to the specific lateralized mechanism(s) of attentional control. In fact, in the former case, we should not expect any reversal of asymmetries. Thus, we compared the Simon effect of right-handed subjects with that of left-handed subjects.

\section{EXPERIMENT 2}

\section{Method}

Subjects. Twenty-four students of the University of Padua (2332 years of age) participated in the experiment. Half of the subjects ( 6 male and 6 female) were right-handed, and the other half ( 6 male and 6 female) were left-handed. Handedness was measured with the Edinburgh Handedness Inventory (Oldfield, 1971). A criterion of $90 \%$ was used for inclusion in the right- or the left-handedness group. All the subjects had normal or corrected-to-normal vision and were unaware of the purpose of the experiment.

Apparatus, Stimuli, and Procedure. The apparatus, experimental setting, and procedure were as in Experiment 1.

\section{Results and Discussion}

Correct RTs (excluding anticipations - i.e., RTs shorter than $150 \mathrm{msec}$ ) and choice errors were submitted to ANOVAs. The ANOVA on correct RTs had one between- subjects factor (handedness) and two within-subjects factors (stimulus position and response position).

No main effect approximated significance. The interaction between handedness and stimulus position did approximate significance $\left[F(1,22)=3.82, M S_{\mathrm{e}}=246.21\right.$, $M S$ effect $=940.38, p=.06]$. The left-handed subjects tended to respond faster to left than to right stimuli (469 vs. $475 \mathrm{msec}$ ), whereas the right-handed subjects tended to respond faster to right than to left stimuli (478 vs. $484 \mathrm{msec})$.

The interaction between handedness and response position was significant $\left[F(1,22)=4.42, M S_{\mathrm{e}}=865.29, M S\right.$ effect $=3,825.12, p<.05]$. The left-handed subjects responded faster with the left than with the right hand (466 vs. $478 \mathrm{msec}$ ), whereas the right-handed subjects were faster with the right hand (475 vs. $488 \mathrm{msec}$ ).

Finally, the interaction between stimulus position and response position was significant $[F(1,22)=18.18$, $M S_{\mathrm{e}}=929.35, M S$ effect $\left.=16,897.49, p<.001\right]$. An overall Simon effect was present: The subjects responded faster with the right hand to right than to left stimuli (463 vs. $490 \mathrm{msec}$ ) and faster with the left hand to left than to right stimuli (464 vs. $490 \mathrm{msec}$ ).

Even though the three-way interaction was not significant, indicating that the Simon effect was reliable in both groups, we analyzed the asymmetries. The handedness $\times$ stimulus position interaction and the handedness $X$ response position interaction indicated that asymmetries had reliably different trends in the two groups. As can be seen in Figure 5, the overall size of the Simon effect did not differ in the two groups: Left-handed subjects showed a 24msec Simon effect, and right-handed subjects showed a 29.5-msec Simon effect. However, the asymmetries were reversed in the two groups.

The stSE for the right-handed group was $17 \mathrm{msec}$ on the left and $42 \mathrm{msec}$ on the right, whereas that for the left-

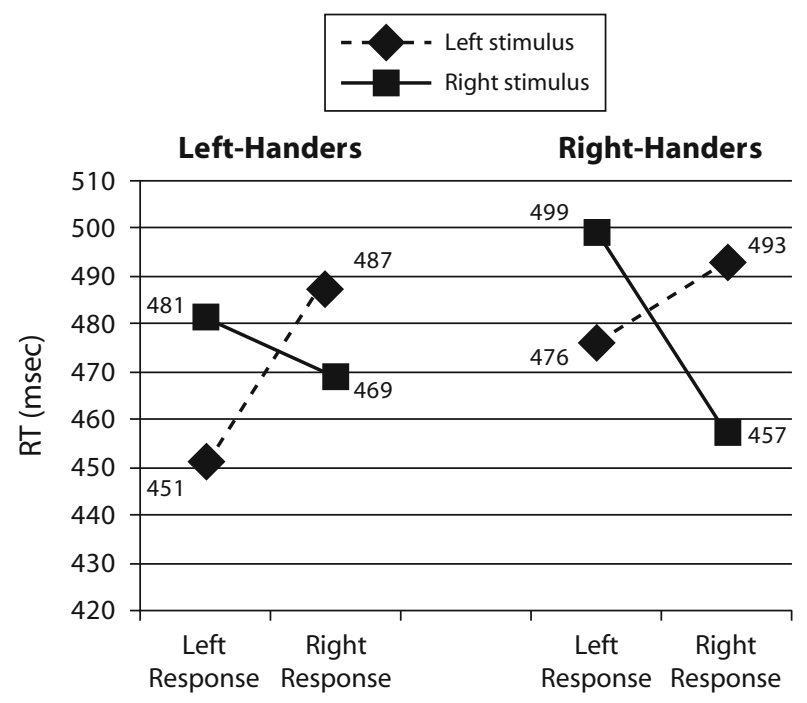

Figure 5. Mean reaction times for the three-way interaction showing the reversed pattern of asymmetries in the left-handed subjects of Experiment 2 of the present study. 
handed group was $36 \mathrm{msec}$ on the left and $12 \mathrm{msec}$ on the right. The reSE showed a tendency in the same direction, the interaction being only marginally significant. In the right-handed group, reSE was $23 \mathrm{msec}$ on the left and $36 \mathrm{msec}$ on the right, whereas in the left-handed group it was $30 \mathrm{msec}$ on the left and $18 \mathrm{msec}$ on the right.

It can be objected that the handedness $\times$ stimulus position interaction only approaches significance. We dealt with this problem through orthogonal planned comparisons (Plans 2 and 3 ) and found that for the left-handed subjects the left reSE did reach significance $[F(1,11)=5.71$, $M S_{\mathrm{e}}=954.16, M S$ effect $\left.=5,447.20, p=.0359\right]$ whereas the right reSE did not $\left[F(1,11)=4.30, M S_{\mathrm{e}}=451.25, M S\right.$ effect $=1,941.66, p=.0623]$. In addition, the left stSE reached significance $\left[F(1,11)=5.00, M S_{\mathrm{e}}=1,596.93\right.$, $M S$ effect $=7,986.57, p=.0470]$ whereas the right stSE did not $\left[F(1,11)=0.79, M S_{\mathrm{e}}=1,018.59, M S\right.$ effect $=$ $812.36, p=.3909]$. In the right-handed group, neither the left reSE $\left[F(1,11)=4.37, M S_{\mathrm{e}}=698.07, M S\right.$ effect $=$ $3,053.72, p=.0605]$ nor the left stSE $[F(1,11)=3.10$, $M S_{\mathrm{e}}=506.57, M S$ effect $\left.=1,571.40, p=.1059\right]$ reached significance. As usual, both the right reSE $[F(1,11)=$ $30.46, M S_{\mathrm{e}}=247.64, M S$ effect $\left.=7,543.05, p=.0002\right]$ and the right stSE $\left[F(1,11)=22.47, M S_{\mathrm{e}}=467.20, M S\right.$ effect $=10,500.17, p=.0006]$ reached significance.

The ANOVA on errors, identical to that performed on RTs, showed a significant interaction only between stimulus position and response position $[F(1,22)=8.84, p<$ $.01]$. Error rates were $0.5 \%$ for both corresponding left responses and corresponding right responses, $1.4 \%$ for noncorresponding left responses, and $1 \%$ for noncorresponding right responses. Since no other main effects or interactions reached significance, it can be concluded that there is no evidence of asymmetries on error data.

\section{CONCLUSIONS}

In the present article, we addressed the problem of leftright asymmetries in the Simon effect. Until now, such asymmetries had attracted very little attention. Our first goal was to provide a method for investigating asymmetries and for estimating the presence, size, and direction of the asymmetries that can be found in the literature.

We started by examining typical statistical analyses of the results in Simon task studies and pointed out that the interaction-based ANOVA designs, in which information about the two sides of space are kept separated, did allow one to assess the presence of asymmetries to some extent. In contrast, the more recent practice of collapsing left and right responses and stimuli into a single "correspondence" factor hides the asymmetries.

By way of new analyses of some of our previously published and unpublished data, we have shown that asymmetries might be present in the stSE, in the reSE, or in both. In addition, we have shown that left and right Simon effects can be of different sizes and both significant; one of them (almost always the left) can be smaller and nonsignificant, and the other, regular and significant; or they can both be small and nonsignificant. In two examples, the left Simon effect, although approximately $20 \mathrm{msec}$, was significantly smaller than the right Simon effect (Tagliabue et al., 2002; Tagliabue et al., 2000). In another example, the left stSE was $9 \mathrm{msec}$, the left reSE was $11 \mathrm{msec}$, and neither was significant. Under these circumstances, it is difficult to draw conclusions on the basis of the overall Simon effect. There are even examples in the literature in which the left Simon effect was actually reversed. We claim that the type of analysis employed might be crucial, as attested by the fact that in some influential studies (Hedge \& Marsh, 1975; Simon et al., 1981) asymmetries were evident but went completely unnoticed, thus remaining unexplained.

Our next step was to clarify the relations between means and statistical values of the response position factor and the stSE, and between means and statistical values of the stimulus position factor and the reSE. It emerged that a shorter RT on one side is necessarily accompanied by a greater stSE on the same side, and an RT advantage for a stimulus on one side is necessarily accompanied by a greater reSE on the same side. That is, the difference between RTs to the left stimuli and RTs to the right stimuli is half the difference between the right reSE and the left reSE, and, conversely, the difference between the left and right response RTs is half the difference between the right reSE and the left reSE.

These relations are important because they provide a tool to carry out a review of the literature on the asymmetries in the Simon effect, even if these asymmetries are not explicitly reported. Moreover, they might render it possible to conduct a meta-analysis on asymmetries regardless of whether they are reported or not. A review of the literature would be important to quantify the presence of asymmetries in experiments in which Simon tasks were employed and to determine the direction of such asymmetries. Very importantly, the statistical relations just mentioned would allow us to infer, from previous studies in which no direct reference to asymmetries was made, whether or not asymmetries were present and, if so, their direction. In the examples provided above, asymmetries very often showed a smaller left Simon effect (either stSE or reSE; Craft \& Simon, 1970; Simon, 1968; Simon et al., 1973; Simon \& Rudell, 1967; Simon et al.,1970; Wallace, 1971). It would be very interesting to figure out why a reverse pattern of asymmetries sometimes manifests itself, as in the studies by Callan et al. (1974) and Hommel (1993, 1996).

We then presented the results of a new experiment and showed that the asymmetries might be so strong as to dramatically reduce the overall Simon effect to a nonsignificant level. This is certainly an extreme case, since usually a global Simon effect is found. Nevertheless, these data led us to propose a new method for investigating the Simon effect, which might provide additional information about asymmetries. This new method would be that of orthogonal planned comparisons, which, thanks to a different form of variance partitioning, is more informative than more traditional methods. Orthogonal planned comparisons provide more conclusive information in the sense that they indicate not only whether there is an asymmetry in the size of the Simon effect on the two sides, but also whether any such asymmetry is significant or not.

Finally, we reasoned that the possible causes of asymmetries could be the lateralization of the processes im- 
plied in the Simon task - that is, response selection and attention. By considering evidence in favor of the left hemisphere's specialization for both selection of action and attention in right-handed subjects, we predicted a reversal of asymmetries (or a more symmetric Simon effect) in left-handed subjects. To test this prediction, we carried out Experiment 2, in which we compared the Simon effect of left- and right-handed subjects. Indeed, we found a reversed pattern of Simon effect asymmetries in the lefthanded subjects, thus confirming the stronger version of our hypothesis.

Note that the prediction was formulated within the framework of the attentional shift hypothesis (Stoffer, 1991; Umiltà \& Nicoletti, 1992) discussed in the introduction, which, in accordance with the widely accepted idea that the Simon effect occurs at the response selection stage, provides an explanation of why the stimulus position code is formed. Is this prediction, and the data that confirm it, in line with the referential coding hypothesis too?

The referential coding hypothesis states that the spatial code is formed with reference to the frame in which the stimulus is embedded, independently of attentional movements (Hommel, 1993). On the other hand, the referential coding hypothesis predicts that the positional code facilitates the corresponding response because of its similarity to the response code (Hommel, 1993). It is not likely that the similarity between the left stimulus and the left response is smaller than the similarity between the right stimulus and the right response (or vice versa, depending on the handedness of the subject). Hence, there is no apparent reason for which the advantage of the corresponding response should be smaller on one side than on the other. In other words, it seems that the presence of asymmetries casts doubt on the referential coding hypothesis or, at least, leads to the need for an improvement of the theory that would help explain why the Simon effect is not symmetric.

Certainly, no comparisons between theories or improvements are possible in the absence of adequate statistical tools. With the present methodological contribution, we have attempted to provide such a tool. The most important implication of this study, however, is that, in future research, a direct and systematic statistical investigation of asymmetries in the reSE or the stSE should be conducted through the use of orthogonal planned comparisons. Without a doubt, collapsing left and right RTs into the single factor of "correspondence" should be avoided.

\section{AUTHOR NOTE}

This work was supported by Grant PRIN 2003112997_002 from MIUR to C.U. Correspondence concerning this article should be addressed to M. Tagliabue, Dipartimento di Psicologia Generale, Università di Padova, via Venezia 8, 35131 Padova, Italy (e-mail: mariaelena .tagliabue@unipd.it).

\section{REFERENCES}

BradshaW, J. L. (1989). Hemispheric specialization and psychological function. New York: Wiley.

Callan, J., Klisz, D., \& Parsons, O. A. (1974). Strength of auditory stimulus-response compatibility as a function of task complexity. Journal of Experimental Psychology, 102, 1039-1045.

Craft, J. L., \& Simon, J. R. (1970). Processing symbolic information from a visual display: Interference from an irrelevant directional cue. Journal of Experimental Psychology, 83, 415-420.

De Gonzaga Gawryszewski, L., Riggio, L., Rizzolatti, G., \& UMILTÀ, C. (1987). Movements of attention in the three spatial dimensions and the meaning of "neutral" cues. Neuropsychologia, 25, 19-29.

Downing, C. J., \& PINKER, S. (1985). The spatial structure of visual attention. In M. I. Posner \& O. S. M. Marin (Eds.), Attention and performance XI (pp. 171-187). Hillsdale, NJ: Erlbaum.

Hedge, A., \& MARSH, N. W. A. (1975). The effect of irrelevant spatial correspondence on two-choice response-time. Acta Psychologica, 39, 427-439.

Hommel, B. (1993). The role of attention for the Simon effect. Psychological Research, 55, 208-222.

Hommel, B. (1996). S-R compatibility effects without response uncertainty. Quarterly Journal of Experimental Psychology, 49A, 546-571.

Hommel, B., \& LiPPA, Y. (1995). S-R compatibility effects due to context-dependent spatial stimulus coding. Psychonomic Bulletin \& Review, 2, 370-374.

Keppel, G. (1991). Design and analysis: A researcher's handbook (3rd ed.). Englewood Cliffs, NJ: Prentice Hall.

Lu, C.-H., \& Proctor, R. W. (1995). The influence of irrelevant location information on performance: A review of the Simon and spatial Stroop effects. Psychonomic Bulletin \& Review, 2, 174-207.

Masaki, H., Takasawa, N., \& YamazaKi, K. (2000). An electrophysiological study of the locus of the interference effect in a stimulusresponse compatibility paradigm. Psychophysiology, 37, 464-472.

Nicoletti, R., \& Umiltà, C. (1994). Attention shifts produce spatial stimulus codes. Psychological Research, 56, 144-150.

OldFIELD, R. C. (1971). The assessment and analysis of handedness: The Edinburgh inventory. Neuropsychologia, 9, 97-113.

Proctor, R. W., \& Lu, C.-H. (1999). Processing irrelevant location information: Practice and transfer effects in choice-reaction tasks. Memory \& Cognition, 27, 63-77.

Rushworth, M. F. S., Nixon, P. D., Renowden, S., Wade, D. T., \& Passingham, R. E. (1997). The left parietal cortex and motor attention. Neuropsychologia, 35, 1261-1273.

Schluter, N. D., Krams, M., Rushworth, M. F. S., \& Passingham, R. E. (2001). Cerebral dominance for action in the human brain: The selection of actions. Neuropsychologia, 39, 105-113.

Simon, J. R. (1968). Effect of ear stimulated on reaction time and movement time. Journal of Experimental Psychology, 78, 344-346.

Simon, J. R., Craft, J. L., \& Webster, J. B. (1973). Reactions toward the stimulus source: Analysis of correct responses and errors over a five-day period. Journal of Experimental Psychology, 101, 175-178.

Simon, J. R., \& Rudell, A. P. (1967). Auditory S-R compatibility: The effect of an irrelevant cue on information processing. Journal of Applied Psychology, 51, 300-304.

SimON, J. R., SLy, P. E., \& VilapakKam, S. (1981). Effect of compatibility of S-R mapping on reactions toward the stimulus source. Acta Psychologica, 47, 63-81.

Simon, J. R., Small, A. M., Ziglar, R. A., \& Craft, J. L. (1970). Response interference in an information processing task: Sensory versus perceptual factors. Journal of Experimental Psychology, 85, 311-314.

STOFFer, T. H. (1991). Attentional focussing and spatial stimulusresponse compatibility. Psychological Research, 53, 127-135.

Tagliabue, M., Zorzi, M., \& Umiltà, C. (2002). Cross-modal remapping influences the Simon effect. Memory \& Cognition, 30, 18-23.

Tagliabue, M., Zorzi, M., Umiltà, C., \& Bassignani, F. (2000). The role of long-term-memory and short-term-memory links in the Simon effect. Journal of Experimental Psychology: Human Perception \& Performance, 26, 648-670.

Umiltà, C. (2000). Visuospatial attention. In F. Boller, J. Grafman, \& G. Rizzolatti (Eds.), Handbook of neuropsychology (2nd ed., Vol. 1, pp. 393-425). Amsterdam: Elsevier.

Umiltì, C., \& LiotTi, M. (1987). Egocentric and relative spatial codes in S-R compatibility. Psychological Research, 49, 81-90.

Umiltì, C., \& Nicoletti, R. (1985). Attention and coding effects in S-R compatibility due to irrelevant spatial cues. In M. I. Posner \& 
O. S. M. Marin (Eds.), Attention and performance XI (pp. 457-471). Hillsdale, NJ: Erlbaum.

Umiltà, C., \& Nicoletti, R. (1992). An integrated model of the Simon effect. In J. Alegria, D. Holender, J. Junça de Morais, \& M. Radeau (Eds.), Analytic approaches to human cognition (pp. 331-350). Amsterdam: North-Holland.

WALLACE, R. J. (1971). S-R compatibility and the idea of a response code. Journal of Experimental Psychology, 88, 354-360.

\section{NOTES}

1. Note that here we will use these data to better explain our points. We will consider further data taken from the literature in the next section.
2. Data analyses were carried out with the R Project software.

3. We consider only the condition in which stimuli and responses are both arranged horizontally and the stimuli are of the same color, because it is the condition most similar to that of the regular Simon task that is the object of the present investigation. In addition, this was the condition with the smallest influence from other potentially interfering factors.

4. These data are inferred from the figure because they are not mentioned in the text.

(Manuscript received June 14, 2005;

revision accepted for publication November 29, 2005.) 